\title{
Investigating Treatment of Primary Axillary Hyperhidrosis With a Topical Retrometabolic Anticholinergic Drug
}

Nicholas Bodor, PhD', Adam Friedman, MD², Stacy Smith, MD³, James Del Rosso, MD4, Janet Dubois, MD ${ }^{5}$, Adelaide Hebert, MD',

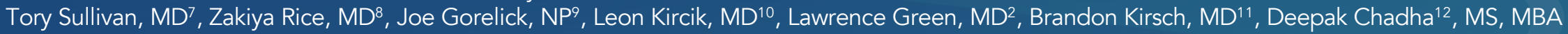

\section{Background} Dermatology, Emory University School of Medicine, Atlanta, GA,
Dermatology, Naples, FL, '2Brickell Biotech, Inc., Boulder, CO

Anticholinergic medications are useful for the management of primary axillary hyperhidrosis (AHH). However, due to inhibition of cholinergic receptors in tissues other than the targeted sweat glands, the use of both oral and topical anticholinergics for the treatment of AHH often results in systemic side effects, such as dry mouth, blurred vision, mydriasis, and urinary hesitation. (rostrual ogue of glycopyrrolate.

\section{Objective}

A need exists to find new anticholinergics with maximal therapeutic benefits and minimal systemic side effects for the treatment of $\mathrm{AHH}$. Retrometabolic drug design is intended to create drugs with an increased therapeutic index by integrating metabolism AHH. Retrometabolic drug design is intended
considerations into the drug design process. ${ }^{1}$

\section{Methods}

Unlike glycopyrrolate, SB includes a readily hydrolyzable ester moiety. To obtain this metabolically sensitive site, the "inactive metabolite" approach to retrometabolic drug design was used. 1 A hydrolytically sensitive function was introduced in a remote site of the molecule, away from the pharmacophore. ${ }^{2}$ The molecular structure was further refined based on binding to the relevant muscarinic receptors, and the most potent optical isomer was selected. ${ }^{3}$ The specific enzyme responsible for hydrolytic metabolism of SB was identified. ${ }^{4}$ Here we review existing pharmacokinetic and pharmacodynamic data for the retrometabolically designed investigational drug $S B$

\section{Results}

Binding studies at human muscarinic receptors (M1-M4) and guinea-pig ileum assays have found SB to have potency close to glycopyrrolate with a shorter duration of action. ${ }^{2}$ The mydriatic effects of SB were compared to those of glycopyrrolate in rabbits, ${ }^{2}$ human port fold suprathe fold suprathare $\mathrm{ng} / \mathrm{mL}$ and many results below the limit of quantitation. 5 To date, more than 1300 subiects have been exposed to $\mathrm{SB}$ across various clinical studies, and $<5 \%$ subjects have been discontinued due to adverse events.

\section{Conclusion}

SB is an anticholinergic agent synthesized according to the principles of retrometabolic drug design. Both nonclinical and clinical studies support the concept that the retrometabolic design of SB may confer certain safety and PK benefits without compromising
desired anticholinergic effect.

\section{Sofpironium Bromide Comparison to Glycopyrrolate}

Once in circulation, sofpironium bromide rapidly hydrolyzes into its metabolite (BBI-4010), a less active anticholinergic agent, therefore reducing its potential for side effects

Glycopyrrolate

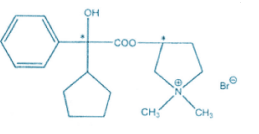

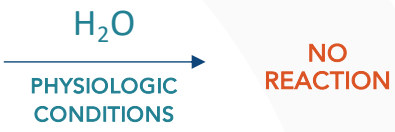

Sofpironium Bromide

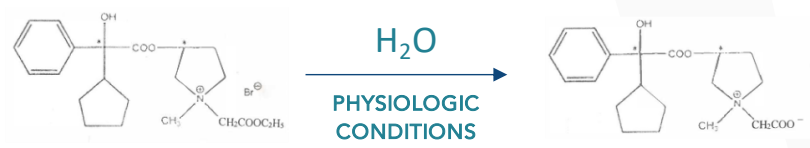

BBI-4010

\section{Funding Statement}

Brickell Biotech, Inc. supported the study and preparation of this abstract.

\section{References}

Bodor N, Buchwald P. Retrometabolic Drug Design and Targeting. Wiley; 2013

$2 \mathrm{Ji}$ F, Wu W, Dai X, et al. Synthesis and pharmacological effects of new, N-substituted soft anticholinergics based on glycopyrrolate. J Pharm

"Tóth-Sarudy E, Tóth G, Pallagi I, et al. Preparation and biological effects of pure stereoisomeric novel soft anticholinergics. Pharm. 2006;661(2):90-96. 4 Samir A, Ohura K, Bodor N, Imai T. Identification of Major Esterase Involved in Hydrolysis of Soft Anticholinergic (2R3'R-SGM) Designed From

5Kirsch B. An Open-Label, Repeat-Dose, Maximum-Use Study to Evaluate the Pharmacokinetics of Sofpironium Bromide Gel, $15 \%$ Topically Applied 